\title{
Mullerian Aplasia and Hyperandrogenism
}

National Cancer Institute

\section{Source}

National Cancer Institute. Mullerian Aplasia and Hyperandrogenism. NCI Thesaurus.

Code C120376.

Deficiency of the glycoprotein WNT 4, associated with loss of function mutation(s) in the WNT 4 gene. The condition in $46, X X$ individuals is characterized by mild hyperandrogenism, absence of underdevelopment of the uterus, and sometimes absence of underdevelopment of the vagina. 